\title{
LARGE-EDDY SIMULATION OF OSCILLATORY FLOW, SEDIMENT TRANSPORT AND MORPHODYNAMICS OVER RIPPLES
}

\author{
Georgios Leftheriotis, University of Patras, gleytheriot@upatras.gr \\ Athanassios Dimas, University of Patras, adimas@upatras.gr
}

\begin{abstract}
In the present study, a well-resolved large-eddy simulation was coupled to a morphodynamical model in order to study the sediment dynamics induced by an oscillatory flow over a sandy bed, as well as the creation/evolution of ripples under hydrodynamic forcing. The simulations were based on the numerical solution of the Navier-Stokes equations for the flow, empirical formulas for the bed load, and the advection-diffusion equation for the suspended sediment. The evolution of the bed form was obtained by the numerical solution of the conservation of sediment mass equation. The Immersed Boundary method was implemented for the imposition of fluid and sediment boundary conditions on the moving bed surface. The model was effectively validated against laboratory measurements. Results are presented for ripple creation and propagation from a quasi-flat bed, as well as results of ripples adapting to water conditions.
\end{abstract}

\section{INTRODUCTION}

Interaction of wave-induced oscillatory flows with the sandy bottom leads to the modification of the bed surface and the generation of coherent small-scale bed forms, generally known as ripples. The geometry of these structures has a strong impact on the waveinduced bottom boundary layer processes, which control sediment transport in coastal areas. Thus, accurate predictions of the ripple shape under certain flow conditions are extremely important in coastal management. During the last decades, significant progress has been made in understanding bed form dynamics. Due to the increased computer power, numerical modeling nowadays captures not only the characteristics of the flow field but also those of turbulence, including the presence of coherent structures which is the main characteristic of oscillatory flows above rippled beds. Marieu et al. (2008) developed a morphological module in order to perform numerical simulations of vortex ripple growth and evolution. They presented a morphologic approach based on a modified non-oscillatory central scheme for ripple growth and slope avalanching, showing that the bed tends to reach the same equilibrium shape regardless of the initial bed shape. Kraft et al. (2011) calculated numerically the suspended sediment transport as well as the movement of a ripple structure in a channel flow using large-eddy simulation (LES). The motion of the interface between fluid and sediment bed was captured by the level-set method. Nabi et al. (2013) presented a 3D numerical model for morphodynamic processes on relatively small temporal and spatial scales using LES. In the present work, LES is coupled to a morphodynamic model is being presented for the study of ripple creation and growth, as well as ripples adapting to changing water conditions. The evolution of the bed form was obtained by the numerical solution of the conservation of sediment mass equation (Exner equation). A fractional time-step scheme was used for the temporal discretization, while finite differences were used for the spatial discretization on a Cartesian grid. The Immersed Boundary method was implemented for the imposition of fluid and sediment boundary conditions on the moving bed surface (Balaras, 2004).

\section{RESULTS}

The numerical model was effectively validated against laboratory measurements involving oscillatory wave motion and sediment transport (Fig. 1). Results are presented for ripple creation and propagation from a quasi-flat bed (Fig. 2), as well as results of ripples adapting to water conditions, based on the mobility number, $\psi$ (Fig. 3). The numerical simulations showed that the numerical model was able to capture phenomena of ripple creation and propagation, resulting in ripple dimensions in agreement with those predicted by empirical equations (Nielsen, 1992). As expected, when the simulations were initiated from a random bed form, ripple growth as well as reaching an equilibrium state was faster than the case of initiating from a quasiflat bed. Moreover, it was observed that under the same hydrodynamic forcing, the bed tends to reach the same equilibrium state, regardless of the initial bed form. Finally, re-orientation of ripples was examined, showing that under oscillatory flow conditions, ripples are reoriented perpendicular to the flow direction regardless of their initial formation.

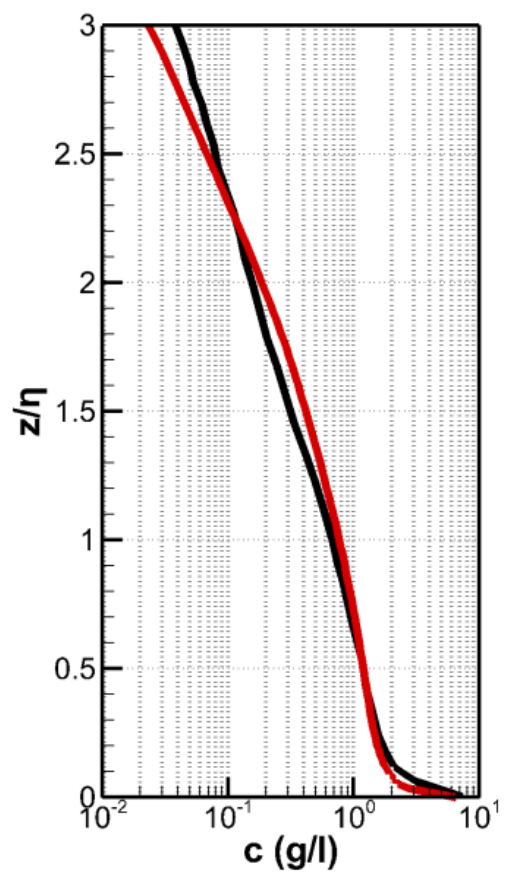

Figure 1 - Time- and bed-averaged concentration of suspended sediment: present numerical simulation (red line) and experimental results (black line) in van der Werf et al. (2007). 

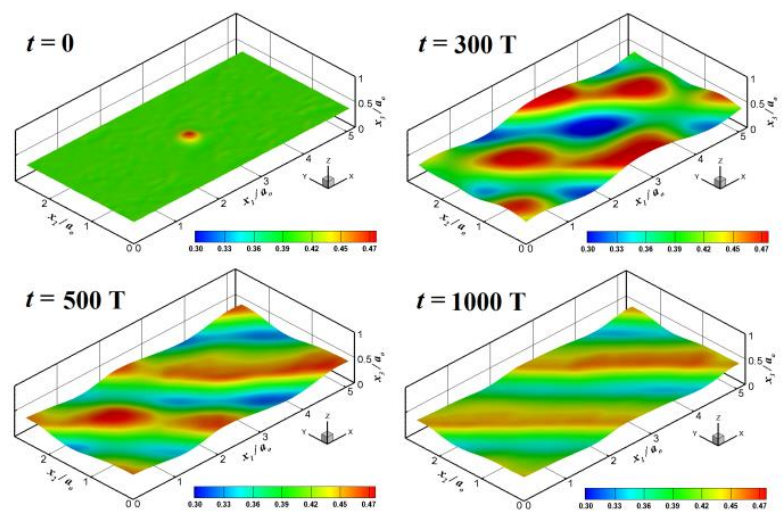

Figure 2 - Ripple creation initiating from a quasi-flat bed, driven by an oblique $\left(45^{\circ}\right)$ oscillatory flow and sediment transport $(\psi=40, \operatorname{Re}=10,000)$.
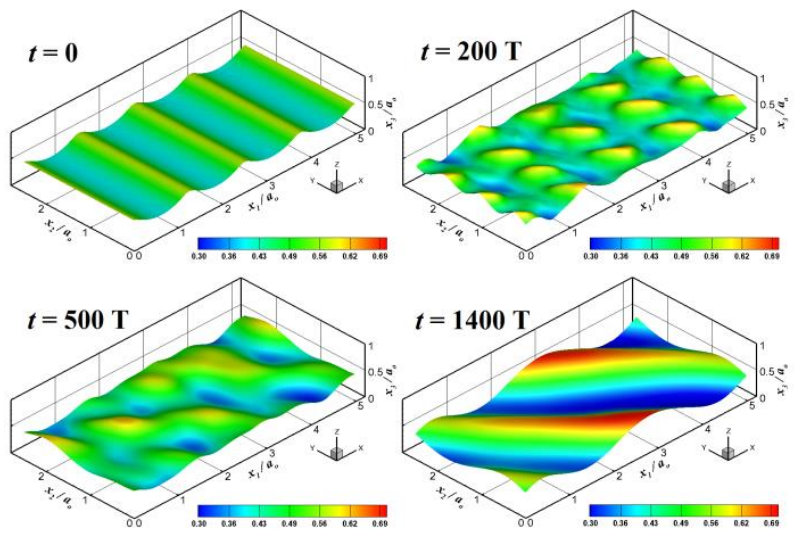

Figure 3 - Ripple re-orientation in time initiating from a rippled bed, driven by an oblique $\left(45^{\circ}\right)$ oscillatory flow and sediment transport $(\psi=40, \operatorname{Re}=10,000)$.

\section{CONCLUSIONS}

LES of oscillatory flow coupled with sediment transport (both bed and suspended load) over a movable bed were performed. Emphasis was placed on the development of a morphodynamic model to perform simulations of the evolution of the bed form. The IB method was employed for the imposition of the fluid and sediment boundary conditions at the rippled bed. Ripple development from quasi-flat or random beds was examined, until the bed form reached an equilibrium shape, adapting to flow conditions based on the mobility parameter, $\psi$. The numerical simulations showed that the morphological model was able to capture physical phenomena of ripple creation, growth, annihilation, merging and migration, resulting in ripple lengths which are in agreement with those predicted by empirical equations. It was also found that under the same hydrodynamic forcing, the bed tends to reach the same equilibrium state, regardless of the initial bed form. The only difference is that the bed reaches the equilibrium state faster when the simulations are initiated from a random bed form than a quasi-flat one. Finally, reorientation of an initially rippled bed was examined, demonstrating that under oscillatory flow conditions, ripples were re-oriented to become perpendicular to the flow direction regardless of their initial formation.

\section{ACKNOWLEDGEMENTS}

This work was funded by the matching contribution (5231) of GSRT to the Initial Training Network
SEDITRANS, implemented within the 7th Framework Programme of the European Commission, and was also supported by computational time granted from the Greek Research \& Technology Network (GRNET) in the National HPC facility - ARIS - under project ID CoastHPC.

\section{REFERENCES}

1. Balaras (2004): Modeling complex boundaries using an external force field on fixed Cartesian grids in large-eddy simulations. Computers \& Fluids, vol. 33, pp. 375-404.

2. Kraft S., Yongqi W., and Oberlack M. (2011): Large eddy simulation of sediment deformation in a turbulent flow by means of level-set method, J. Hydraul. Eng., 137 (11), 1394-1405.

3. Marieu, V., P. Bonneton, D.L. Foster, and F. Ardhuin. (2008): Modeling of vortex ripple morphodynamics, J. Geophys. Res., 113, C09007.

4. Nabi M., de Vriend HJ., Mosselman E., Sloff CJ. and Shimizu Y. (2012): Detailed simulation of morphodynamics: 2. Sediment pickup, transport and deposition, Water Resour. Res., 49.

5. Nielsen (1992): Coastal Bottom Boundary Layers and Sediment Transport. World Scientific, 324pp.

6. van der Werf, Doucette, O' Donoghue and Ribberink (2007): Detailed measurements of velocities and suspended sand concentrations over full-scale ripples in regular oscillatory flow, J. Geophys. Res., vol. 112, F02012. 